\title{
DOS NUEVAS ESPECIES DE ASTEROCHERES BOECK, 1860 (COPEPODA: SIPHONOSTOMATOIDA) DE CUBA
}

\author{
Carlos Varela \\ Acuario Nacional de Cuba (ANC), Calle 1ra \#6002 e/e 60 y 62, C. P. 11300, Playa, Ciudad de La Habana, \\ Cuba.varela06@gmail.com
}

\section{RESUMEN}

Se describen dos especies de copépodos pertenecientes al género Asterocheres Boeck, 1860, los cuales fueron hallados asociados a esponjas del género Agelas Duchassaing y Michelotti, 1864. Se ofrecen además las diferencias más importantes entre las especies nuevas y las más similares.

Palabras clave: Asterocheridae, Siphonostomatoida, Copepoda, Asterocheres, Cuba.

\section{ABSTRACT}

Two new species of copepods belonging to the genus Asterocheres Boeck, 1860 are described. They were collected in association with sponges of the genus Agelas Duchassaing y Michelotti, 1864. The most important differences between those new species and the more similar ones are also given.

Key words: Asterocheridae, Siphonostomatoida, Copepoda, Asterocheres, Cuba.

\section{INTRODUCCIÓN}

Estudios recientes, encaminados a conocer la diversidad de los copépodos sifonostomatoides que viven asociados a invertebrados marinos bentónicos en el archipiélago cubano, han permitido el hallazgo de varios taxa como Asteropontius mycetophylliae Varela, Ortiz y Lalana, 2005 (Varela et al., 2005b), encontrado asociado a corales, Orecturus ortizi Varela y Lalana, 2007 asociado a octocorales (Varela y Lalana, 2007). Onycocheres alatus Stock y Gooding, 1986 (Varela et al., 2005a), asociado a equinodermos y Scottocheres elongatus (Thompson y A. Scott, 1894), Asterocheres reginae Huys y Boxshall, 1994, A. espinosai Varela, Ortiz y Lalana, 2007 y A. garridoi Varela, Ortiz y Lalana, 2007, asociados a esponjas (Varela et al., 2007a, 2007b y 2008). Revisión de material colectado nos ha permitido encontrar dos especies de copépodos, no descritas previamente, pertenecientes al género Asterocheres, que se presentan a continuación.

\section{MATERIALES Y MÉTODOS}

La colecta fue realizada mediante buceo autónomo (SCUBA). Las esponjas hospedadoras de los copépodos, fueron cubiertas con una bolsa de polietileno, después de lo cual se arrancaron del sustrato, cerrándose rápidamente para evitar la pérdida de los animales. Posteriormente se añadieron gotas de formalina a la bolsa y se sacudió el contenido, que posteriormente fue tamizado y fijado. El material estudiado se encuentra depositado en el Departamento de Colecciones Naturales Marinas, del ANC.

\section{RESULTADOS}

TAXONOMÍA

Asterocheres fernandezmilerai sp. nov.

(Figs 1-3)

Diagnosis. Cono oral no llega a la inserción de la pata 1, artejo libre de la pata 5 con 2 setas, garra del maxilípedo es casi el doble de la longitud del artejo precedente, furca caudal casi tan larga como ancha y palpo mandibular con dos artejos. 


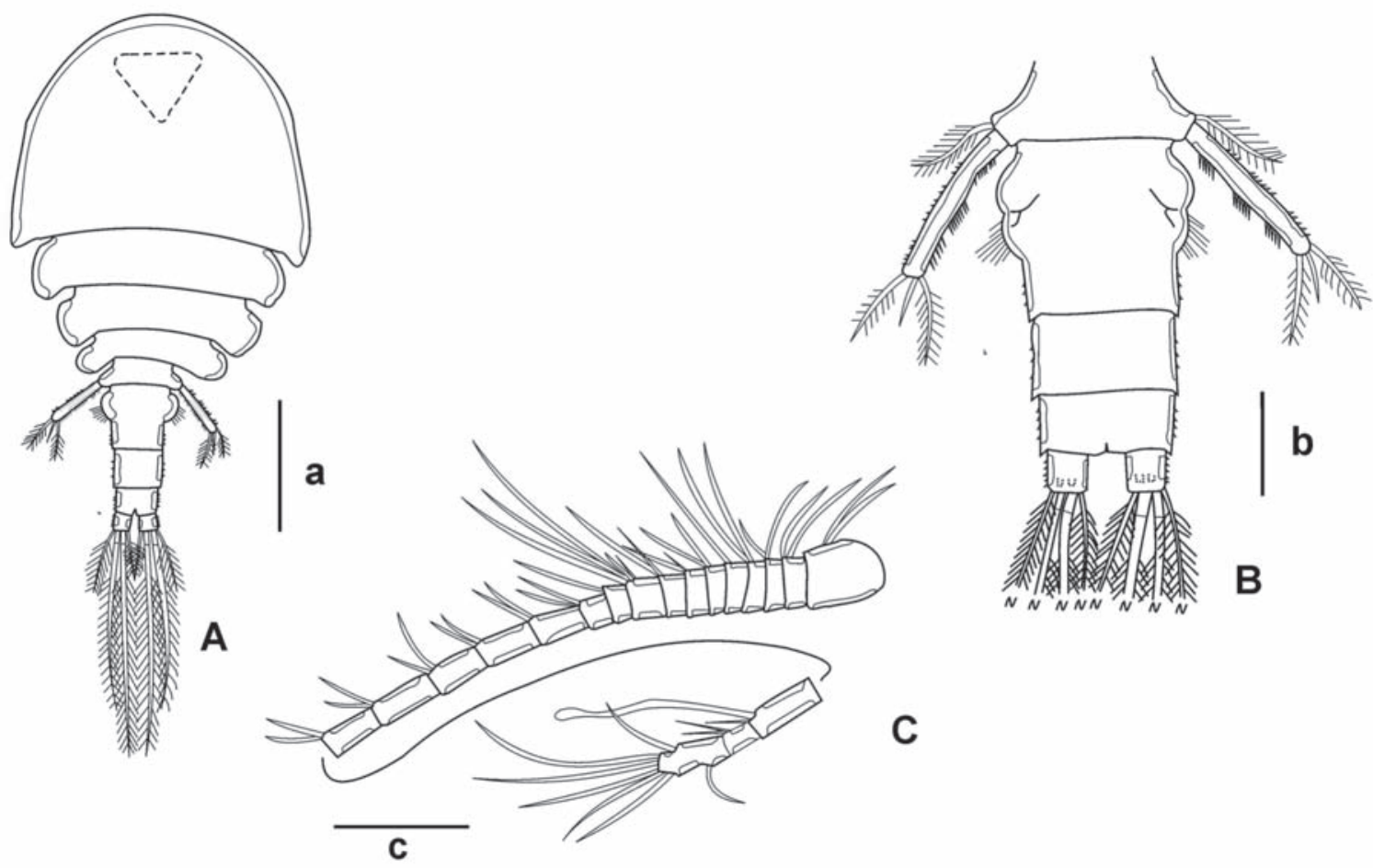

Figura 1. Asterocheres fernandezmilerai sp. nov. (Holotipo). A. Vista dorsal (a); B. Vista dorsal del urosoma (b) y C. Anténula (c). Escala. a: 0, $2 \mathrm{~mm}$; b y c: $0,05 \mathrm{~mm}$.

Diagnosis. Oral cone do not reaches the basal insertion of leg 1, leg 5 with 2 setae, maxiliped claw almost twice the length of preceding segment, caudal furca as long as wide, mandibular palp with 2 articles.

Descripción de la hembra. Largo del cuerpo (excluyendo las setas caudales) $720 \mu \mathrm{m}(725-715 \mu \mathrm{m})$, mayor ancho corporal $400 \mu \mathrm{m}(402-397 \mu \mathrm{m})$, largo del cuerpo 1, 8 veces el ancho (basado en 4 ejemplares). Forma del cuerpo ciclopiforme (Fig. 1A), con prosoma moderadamente alargado, aplanado dorsoventralmente y urosoma cilíndrico. Segmento 1 totalmente fusionado con el cefalosoma, con epímeros redondeados. Segmentos 2 y 3 con casi la misma longitud, casi el mismo largo y con epímeros redondeados. Segmento 4 más estrecho que el segmento que lo precede. Segmento 5 parcialmente cubierto por el segmento que lo precede. Proporción largo: ancho del prosoma 1, 3:1. Proporción entre la longitud del prosoma y la del urosoma 2, 7:1.

Urosoma con 4 segmentos (Fig. 1B). Segmento genital $104 \mu \mathrm{m}$ de largo. Proporción largo: ancho 0,9: 1, redondeado anterolateralmente y con hileras laterales de sétulas. Aberturas genitales en la región más ancha del segmento. Segmento postgenital y anal más anchos que largos (35 x $80 \mu \mathrm{m}$ y $35 \times 73 \mu \mathrm{m})$, proporción largo: ancho 0, 4: 1 en ambos. Rama caudal casi tan larga como ancha, largo es 0,9 veces el ancho, armada con 6 setas.

Anténula (Fig. 1C) $360 \mu \mathrm{m}$ de largo (sin incluir las setas) con 19 artejos. Largo de los artejos, medidos a lo largo de su margen posterior: $26,10,8,10,12,8,10,10,18,10,20,26,24,26,26,32,34$, 18 y $32 \mu \mathrm{m}$, respectivamente. Homologías entre los artejos y setación como sigue: I-3; II-2; III-2; IV2; V-1; VI-2; VII-2; VIII-2; IX-XIII-5; XIV-1; XV-2; XVI-2; XVII-2; XVIII-2; XIX-2; XX-2; XXI-1 + estetasco; XXII-XXIII-2; XXIV-XXVIII-7. Estetasco $14 \mu \mathrm{m}$ de largo. 

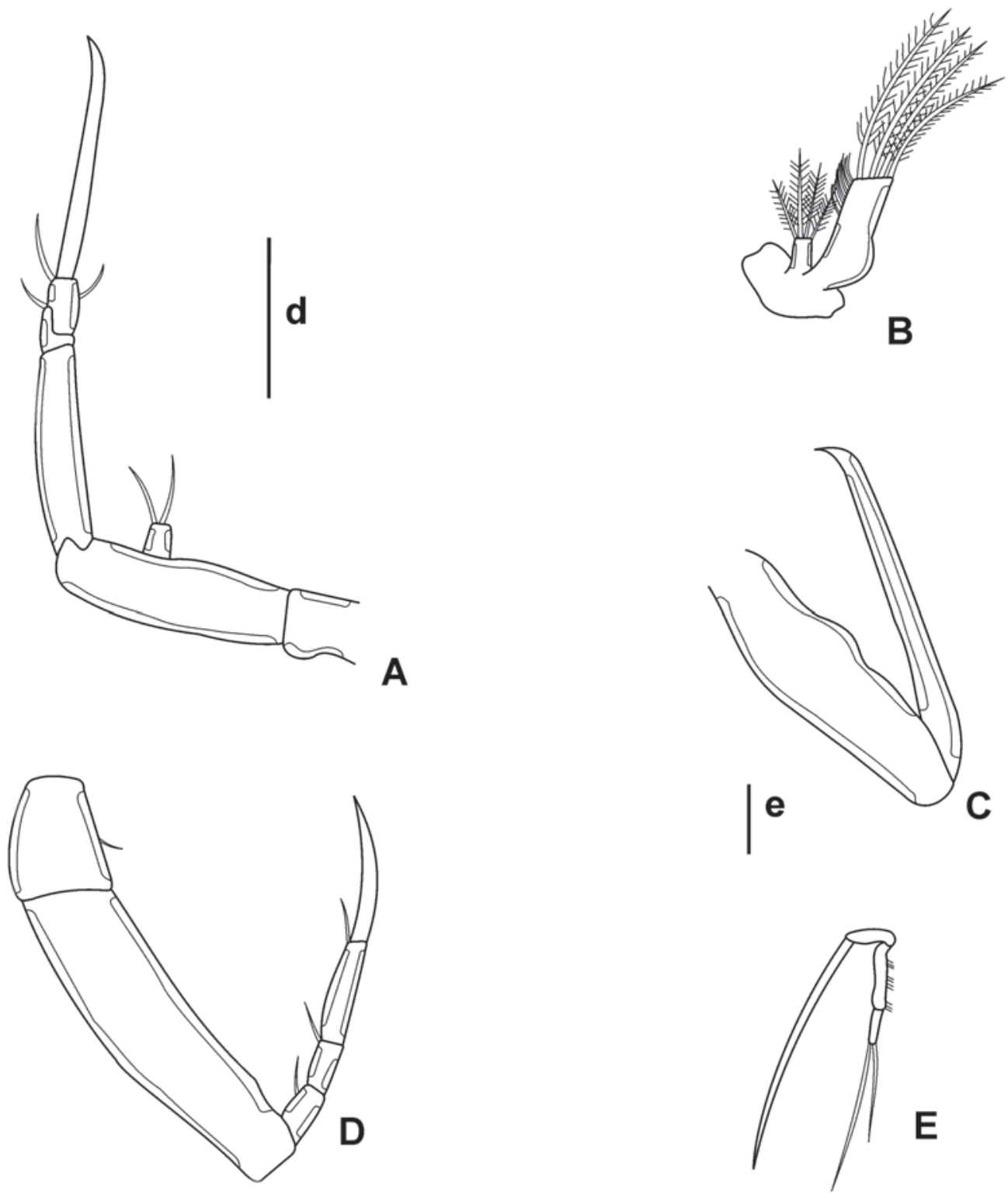

Figura 2. Asterocheres fernandezmilerai sp.nov. (Holotipo). A. Antena (d); B. Maxila (e); C. Maxílula (c); D. Maxilípedo (c) y E. Mandíbula (e). Escala: d, e, 0,05 mm.

Antena (Fig. 2A) $280 \mu \mathrm{m}$ de largo (incluyendo la garra distal), basipodito $80 \mu \mathrm{m}$ de largo, desarmado. Exopodito con un artejo, $17 \mu \mathrm{m}$ de largo, armado con 2 setas distales. Endopodito con 3 artejos; artejo 1, $70 \mu \mathrm{m}$ de largo, desarmado; artejo 2, $8 \mu \mathrm{m}$ de largo, con una seta distal y artejo 3, 19 $\mu \mathrm{m}$ de largo con 2 setas y una garra terminal ligeramente curvada $86 \mu \mathrm{m}$ de largo. Cono oral (Fig. 1A) corto, $86 \mu \mathrm{m}$ de largo. Mandíbula (Fig. 2E) con estilete y palpo mandibular delgado, con 2 artejos; artejo 1 desarmado y artejo 2 con 2 setas.

Maxílula (Fig. 2C) bilobada, ambos lóbulos delgados. Lóbulo interno $55 \mu \mathrm{m}$ de largo, más de dos veces el largo del lóbulo externo, armado con 4 setas distales, margen lateral cubierto con sétulas. Lóbulo externo $22 \mu \mathrm{m}$ de largo, con 4 setas. Maxila (Fig. 2B) con sincoxa de $101 \mu \mathrm{m}$ de largo y la garra distal, $106 \mu \mathrm{m}$ de largo con extremidad curvada. 
Maxilípedo (Fig. 2D) $288 \mu \mathrm{m}$ de largo, con 5 artejos, sincoxa corta, $43 \mu \mathrm{m}$ de largo armada con una pequeña seta en el margen interno; basipodito de $111 \mu \mathrm{m}$ de largo, desarmado; endopodito con 3 artejos, artejos 1 y 2 con casi la misma longitud de $17 \mu \mathrm{m}$ y $12 \mu \mathrm{m}$ respectivamente, y ambos armados con una seta; artejo 3 con $36 \mu \mathrm{m}$ de largo con una seta apical, garra distal de $69 \mu \mathrm{m}$ de largo.

Patas 1 a 4 (Figs. 3 A-D) birramosas, con 3 artejos en cada rama. Todas las setas plumosas y las espinas fuertes y plumosas, excepto la espina distal del artejo 3 del exopodito de la pata 4 que es curvada y lisa en su margen interno. Artejo 1 del exopodito de la pata 1 con una larga espina. La fórmula de la armadura de las patas 1 a 4 es la siguiente:

\begin{tabular}{|ccccc|}
\hline & Coxopodito & Basipodito & Exopodito & Endopodito \\
\hline P1 & $0-1$ & $1-1$ & $\mathrm{I}-1 ; \mathrm{I}-1 ; \mathrm{III}-4$ & $0-1 ; 0-2 ; 1-2-3$ \\
P2 & $0-1$ & $1-0$ & $\mathrm{I}-1 ; \mathrm{I}-1 ; \mathrm{III}-\mathrm{I}-4$ & $0-1 ; 0-2 ; 1-2-3$ \\
P3 & $0-1$ & $1-0$ & $\mathrm{I}-1 ; \mathrm{I}-1 ; \mathrm{III}-\mathrm{I}-4$ & $0-1 ; 0-2 ; 1-1+\mathrm{I}-3$ \\
P4 & $0-1$ & $1-0$ & $\mathrm{I}-1 ; \mathrm{I}-1 ; \mathrm{III}-\mathrm{I}-4$ & $0-1 ; 0-2 ; 1-1+\mathrm{I}-2$ \\
\hline
\end{tabular}

Pata 5 (Fig. 1B) pequeña, $36 \mu \mathrm{m}$ de largo, exopodito largo con 2 setas distales. Segmento 5 presenta una seta cercana a la inserción con el exopodito.
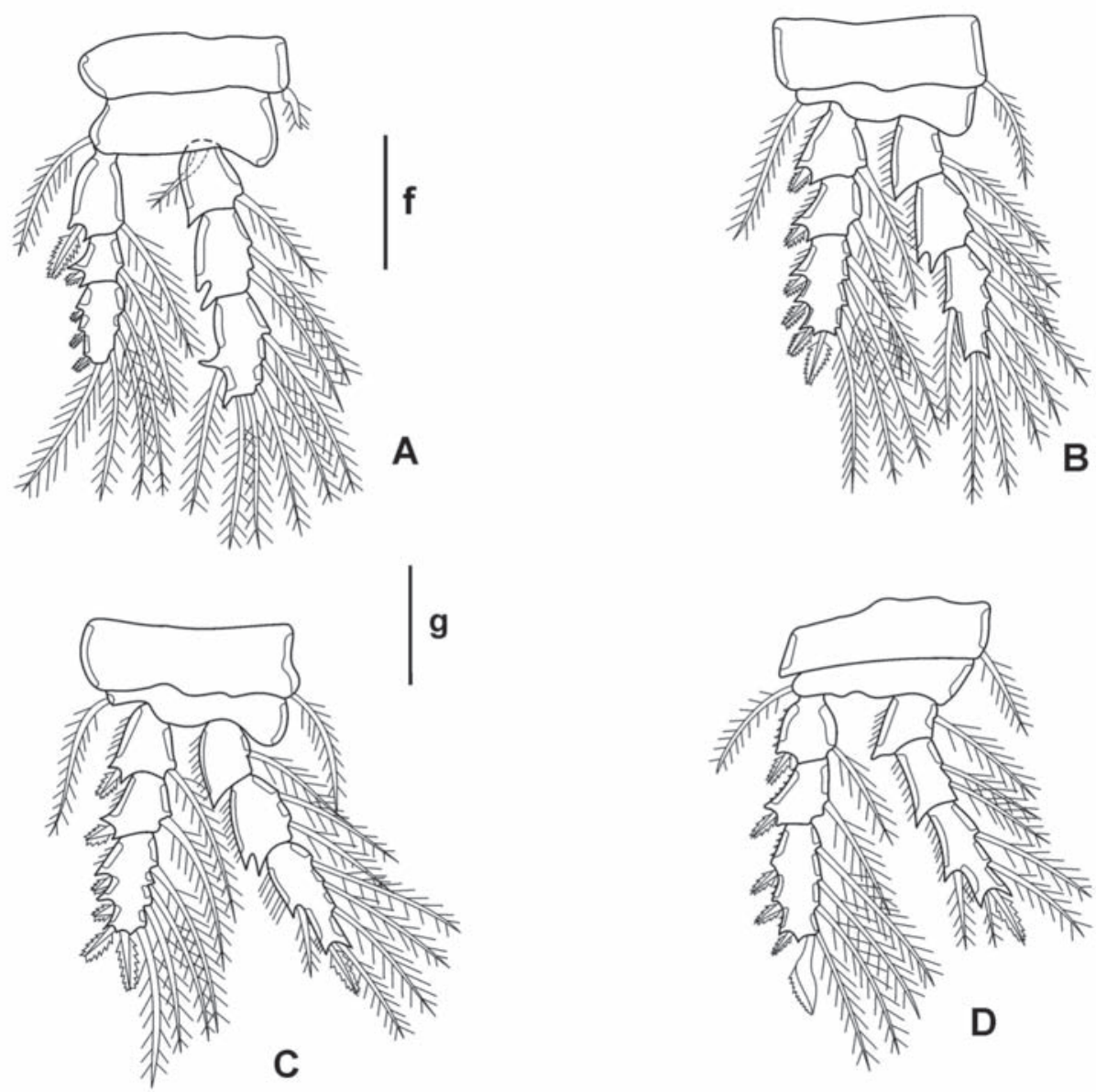

\section{B}

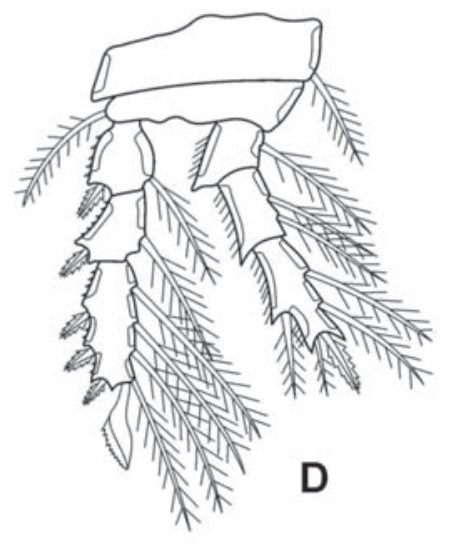

Figura 3. Asterocheres fernandezmilerai sp. nov. (Holotipo). A. Pata 1 (f); B. Pata 2 (g); C. Pata 3 (g) y D. Pata 4 (g). Escala:. f, g: $0,05 \mathrm{~mm}$. 
Tipos. Holótipo: hembra no ovígera. CUBA. Colectados en el sublitoral frente al Acuario Nacional de Cuba, provincia de Ciudad de La Habana, 14. IX. 2008, asociado a la esponja Agelas wiedenmayeri Alcolado, 1984 a $12 \mathrm{~m}$ de profundidad, col. C. Varela. Depositado en ANC 07. 2. 1. 1. 023. Paratipos: 3 hembras. Colectados en la misma fecha y localidad. Depositados junto al holotipo, ANC 07. 2. 1. 1. 024.

Etimología. Esta especie está dedicada a José Fernández Milera destacado malacólogo y maestro de generaciones de biólogos, recientemente fallecido.

Comentario. Asterocheres fernandezmilerai sp. nov., tiene el cono oral que no llega a la inserción de la pata 1 , artejo libre de la pata 5 con 2 setas y la garra del maxilípedo es casi el doble de la longitud del artejo precedente. Dentro del género, solo A. uncinatus (Krigacin, 1873) y A. unicus Johnsson, Rocha y Neves, 2001 comparten estas características. En Asterocheres uncinatus el largo de la furca caudal es el doble del ancho, mientras que en $A$.fernandezmilerai sp. nov., la furca caudal es casi tan larga como ancha. A. unicus tiene el palpo mandibular con solo un artejo mientras que en $A$. fernandezmilerai sp. nov. el palpo mandibular tiene dos artejos.

Asterocheres cubensis sp. nov.

(Figs. 4 y 5)

Diagnosis. Cono oral no llega a la inserción de la pata 1, artejo libre de la pata 5 con 3 setas, rama caudal tan larga como ancha, segmento postgenital casi de la misma longitud que el segmento anal, la garra del maxilípedo es ligeramente de mayor longitud que el artejo que la antecede, anténula con 19 artejos, artejos 1 y 2 del maxilípedo con setas, la pata 5 es casi de la misma longitud que el segmento genital, sincoxa de la maxílula sin estetascos, palpo mandibular con 2 artejos y el exopodito de la antena con 2 setas.

Diagnosis. Oral cone do not reaches the basal insertion of leg 1. Leg 5 with 3 setae. Postgenital and anal segments almost with the same length, maxiliped claw slightly the length of preceding segment, antennules with 19 articles, articles 1 and 2 of maxiliped armed with one setae each, leg 5 almost the same length than genital segment, maxilula's sincoxa witouth aesthetasc, mandibular palp with 2 articles and antenna exopod with 2 setae.

Descripción. Largo del cuerpo (excluyendo las setas caudales) $800 \mu \mathrm{m}(810-790 \mu \mathrm{m})$, mayor ancho corporal $466 \mu \mathrm{m}(471-460 \mu \mathrm{m})$, largo del cuerpo 1, 7 veces el ancho (basado en 2 ejemplares). Forma del cuerpo ciclopiforme (Fig. 4A), con prosoma moderadamente alargado, aplanado dorsoventralmente y urosoma cilíndrico. Segmento 1 totalmente fusionado con el cefalosoma, con epímeros redondeados. Segmentos 2 y 3 con casi la misma longitud y casi el mismo largo y epímeros redondeados. Segmento 4 más estrecho que el segmento que lo precede. Segmento 5 parcialmente cubierto por el segmento que lo precede. Proporción largo: ancho del prosoma 1, 2:1. Proporción entre la longitud del prosoma y la del urosoma 2, 4:1.

Urosoma con 4 segmentos (Fig. 4B). Segmento genital $111 \mu \mathrm{m}$ de largo, largo: ancho ratio 0, 8: 1 , redondeado anterolateralmente y con hileras laterales de sétulas. Aberturas genitales en la región más ancha del segmento. Segmento postgenital más ancho que largo, 52 × $94 \mu \mathrm{m}$; proporción largo: ancho ratio, $0,5: 1$. Segmento anal $52 \mu \mathrm{m}$ x $86 \mu \mathrm{m}, 0,6: 1$. Rama caudal cuadrada $27 \mu \mathrm{m}$, armada con 6 setas.

Anténula (Fig. 4C) $391 \mu \mathrm{m}$ largo (sin incluir las setas) con 19 artejos. Largo de los artejos, medidos a lo largo de su margen posterior: $40,11,8,11,8,8,11,11,17,8,15,26,31,28,35,28,40,17$ y $35 \mu \mathrm{m}$, respectivamente. Homologías entre los artejos y setación como sigue: I-3; II-2; III-2; IV-2; V-1; VI-2; VII-2; VIII-2; IX-XIII-5; XIV-1; XV-2; XVI-2; XVII-2; XVIII-2; XIX-2; XX-2; XXI1+estetasco; XXII-XXIII-2; XXIV-XXVIII-7. Estetasco $108 \mu \mathrm{m}$ de largo. Antena (Fig. 4D) $254 \mu \mathrm{m}$ largo (incluyendo la garra distal), basipodito $81 \mu \mathrm{m}$ de largo, desarmado. Exopodito con un artejo, 17 $\mu \mathrm{m}$ de largo, armado con 2 setas distales. Endopodito con 3 artejos; artejo 1 con $68 \mu \mathrm{m}$ de largo, desarmado; artejo 2 con $6 \mu \mathrm{m}$ de largo, con una seta distal y artejo 3 con $13 \mu \mathrm{m} 2$ setas y una garra terminal ligeramente curvada $86 \mu \mathrm{m}$. Cono oral corto, $73 \mu \mathrm{m}$. 
Mandíbula (Fig. 4E) con estilete y palpo mandibular delgado, con 2 artejos; artejo 1 desarmado y artejo 2 con 2 setas. Maxílula (Fig. 5B) bilobada, ambos lóbulos delgados. Lóbulo interno $60 \mu \mathrm{m}$, más de dos veces el largo del lóbulo externo, armado con 4 setas distales, margen lateral cubierto con sétulas. Lóbulo externo $17 \mu \mathrm{m}$ de largo, con 4 setas. Maxila (Fig. 5A) con sincoxa $102 \mu \mathrm{m}$ de largo y la garra, 119 $\mu \mathrm{m}$ de largo con extremidad curvada. Maxilípedo (Fig. 5C) $238 \mu \mathrm{m}$ de largo, con 5 artejos, sincoxa corta $33 \mu \mathrm{m}$ de largo, armada con una pequeña seta en el margen interno; basipodito $102 \mu \mathrm{m}$ de largo, desarmado; endopodito con 3 artejos, artejos 1 y 2 con casi la misma longitud de $13 \mu \mathrm{m}$ y $15 \mu \mathrm{m}$ respectivamente, y ambos armados con una seta; artejo 3 de $29 \mu \mathrm{m}$ de largo con una seta apical cercana a la garra. Garra curvada, de $46 \mu \mathrm{m}$ de largo.

Patas 1 a 4 (Fig. 5, D-G) birramosas, con 3 artejos en cada rama. Todas las setas plumosas y las espinas fuertes y plumosas, excepto la espina distal del artejo 3 del exopodito de la pata 4 que es curvada y lisa en su margen interno. Artejo 1 del exopodito de la pata 1 con una larga espina. La fórmula de la armadura de las patas 1 a 4 es la siguiente:

\begin{tabular}{|llccc|}
\hline & Coxopodito & Basipodito & Exopodito & Endopodito \\
\hline P1 & $0-1$ & $1-1$ & $\mathrm{I}-1 ; \mathrm{I}-1 ; \mathrm{III}-4$ & $0-1 ; 0-2 ; 1-2-3$ \\
P2 & $0-1$ & $1-0$ & $\mathrm{I}-1 ; \mathrm{I}-1 ; \mathrm{III}-\mathrm{I}-4$ & $0-1 ; 0-2 ; 1-2-3$ \\
P3 & $0-1$ & $1-0$ & $\mathrm{I}-1 ; \mathrm{I}-1 ; \mathrm{III}-\mathrm{I}-4$ & $0-1 ; 0-2 ; 1-1+\mathrm{I}-3$ \\
P4 & $0-1$ & $1-0$ & $\mathrm{I}-1 ; \mathrm{I}-1 ; \mathrm{III}-\mathrm{I}-4$ & $0-1 ; 0-2 ; 1-1+\mathrm{I}-2$ \\
\hline
\end{tabular}

Pata 5 (Fig. 4B) pequeña, $66 \mu \mathrm{m}$ de largo, exopodito largo con 3 setas distales. Segmento 5 presenta una seta cercana a la inserción con el exopodito.
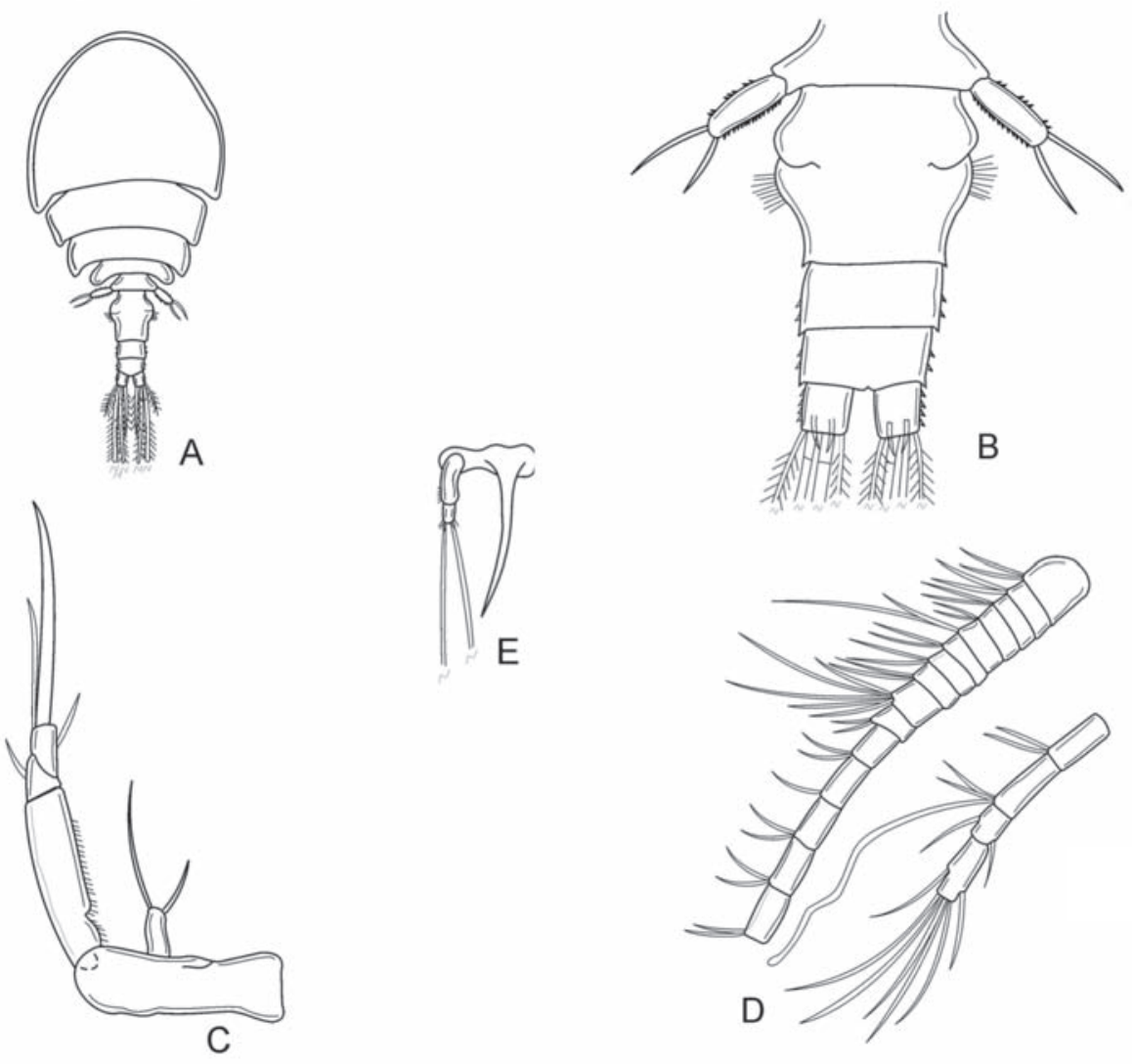

Figura 4. Asterocheres cubensis sp.nov. (Holotipo). A. Vista dorsal (a); B. Vista dorsal del urosoma (d); C. Anténula (c); D. Antena (d) y E. Mandíbula (e). 

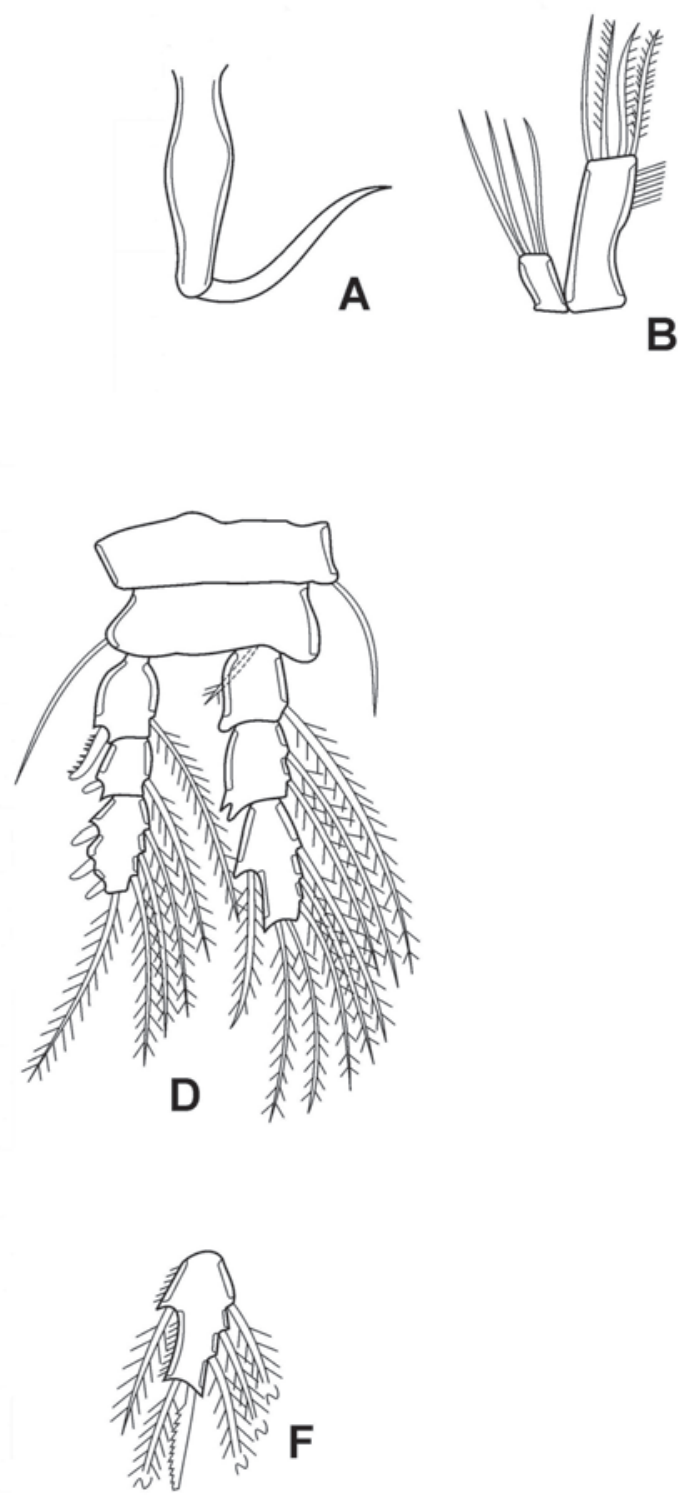

B
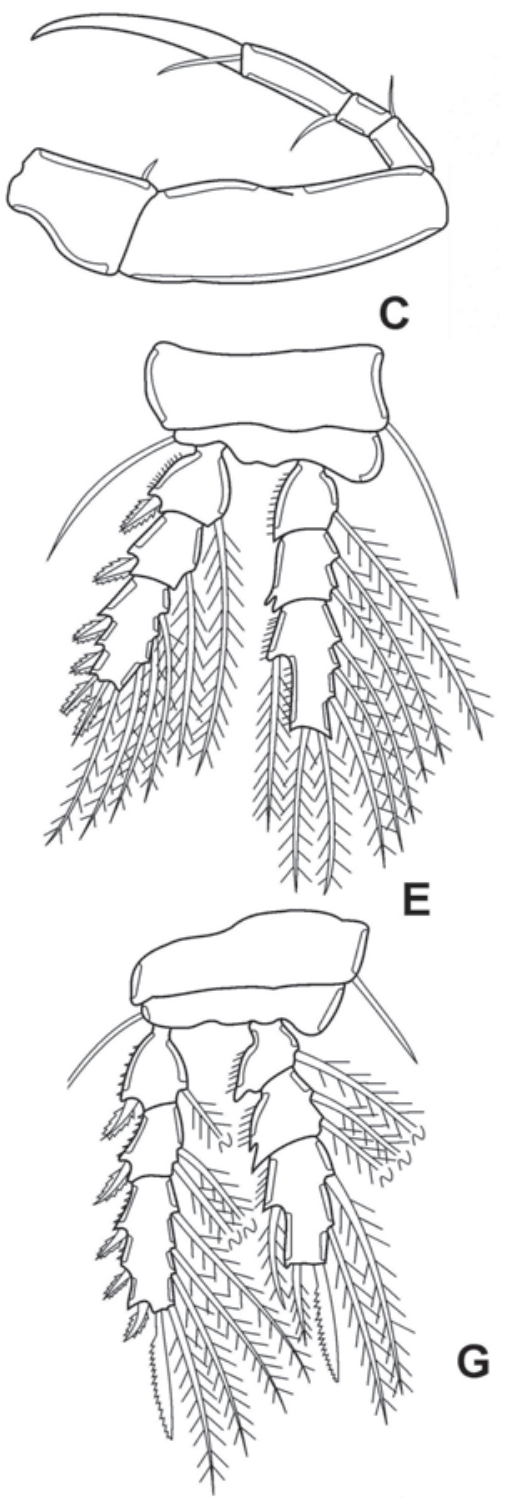

Figura 5. Asterocheres cubensis sp.nov. (Holotipo). A. Maxila (f); B. Maxílula (b); C. Maxilípedo (d); D. Pata 1 (f); E. Pata 2 (g); F. Tercer artejo del exopodito de la pata 3 (g). G. Pata 4 (g).

Tipos. Holótipo: hembra no ovígera. CUBA. Colectado en Playa El Salado, provincia de La Habana, 20. II. 2007, asociado a la esponja Agelas dilatata Duchassaing y Michelotti, 1864 a $18 \mathrm{~m}$ de profundidad, col. C. Varela. Depositado en ANC 07.2. 1. 1. 024. Parátipos: 1 hembra. Colectados en la misma fecha y localidad. Depositados junto al holotipo ANC 07. 2. 1. 1. 025.

Etimología. Esta especie está dedicada a la isla de Cuba, donde fue colectada.

Comentario. Asterocheres cubensis sp. nov. tiene el cono oral que no llega a la inserción de la pata 1, artejo libre de la pata 5 con 3 setas, rama caudal tan larga como ancha, segmento postgenital casi de la misma longitud que el segmento anal, la garra del maxilípedo es ligeramente de más longitud que el artejo precedente y anténula con 19 artejos. Dentro del género solo A. abrohlensis Johnsson, Rocha y Neves, 2001, A. brevisulculus Kim, 2005 y A. walteri Kim, 2004 comparten estas características. 
En Asterocheres abrohlensis los artejos 1 y 2 del endopodito del maxilípedo no presentan setas y la longitud de la pata 5 casi llega a la mitad del segmento genital; mientras que en $A$. cubensis sp. nov., los artejos 1 y 2 del maxilípedo presentan setas y la pata 5 es casi de la misma longitud que el segmento genital. En $A$. brevisulculus el palpo mandibular presenta solo un artejo y el exopodito de la antena tiene 3 setas; mientras que en $A$. cubensis sp. nov., el palpo mandibular presenta 2 artejos y el exopodito de la antena tiene 2 setas. A. walteri tiene un estetasco en la sincoxa de la maxílula y 3 setas en el exopodito de la antena; mientras que $A$. cubensis sp. nov., no presenta estetascos en la sincoxa de la maxílula y el exopodito de la antena presenta 2 setas.

\section{AGRADECIMIENTOS}

Al Sr. Richard Green (Smithsonian Institution, U. S. A.) por la literatura facilitada. A Ernesto Acosta (Acuario Nacional de Cuba, Cuba) por la ayuda en la colecta de los ejemplares.

\section{LITERATURA CITADA}

Varela, C., M. Ortiz y R. Lalana. 2005a. Nuevos registros de copépodos (Crustacea: Maxillopoda: Copepoda), para aguas cubanas. Revista Investigaciones Marinas 26(1): 79-80.

Varela, C., M. Ortiz y R. Lalana. 2005b. Una nueva especie de Asteropontius (Copepoda: Siphonostostomatoida), de Cuba. Solenodon 5: 1-5.

Varela, C., M. Ortiz y R. Lalana. 2007a. Especie nueva de copépodo espongícola (Copepoda: Siphonostomatoida: Asterocheridae), para Cuba. Solenodon 6: 1-7.

Varela, C., M. Ortiz y R. Lalana. 2007b. Una nueva especie de copépodo del género Asterocheres Boeck, 1860 (Copepoda: Siphonostostomatoida), de aguas cubanas. Avicennia 19:31-36.

Varela, C., y R. Lalana. 2007. Especie nueva de Orecturus (Crustacea: Copepoda), para Cuba. Solenodon 6: 15-19.

Varela, C., S. Castellanos y L. Hernández. 2008. Registros nuevos de invertebrados (Cnidaria y Crustacea) para Cuba. Cocuyo 17: 12-14. 